\title{
Physiological Correlates of Aggression and Impulsivity in Free-Ranging Female Primates
}

\author{
Gregory C Westergaard*,', Stephen J Suomi' ${ }^{2}$ Tara J Chavanne', Lisa Houser', Anne Hurley ${ }^{3}$, Allison \\ Cleveland', Philip J Snoy ${ }^{4}$ and J Dee Higley ${ }^{3}$ \\ 'Division of Research and Development, LABS of Virginia, Inc., USA; '2 Laboratory of Comparative Ethology, National Institute of Child Health and \\ Human Development, USA; ${ }^{3}$ Laboratory of Clinical Studies, National Institute of Alcohol Abuse and Alcoholism, USA; ${ }^{4}$ Division of Veterinary \\ Services, Center for Biologics Evaluation and Research, Food and Drug Administration, USA
}

\begin{abstract}
We examined the relations among cerebrospinal fluid (CSF) monoamine metabolite concentrations, plasma hormone concentrations, aggression, and impulsive risk-taking behavior in a free-ranging population of female rhesus macaques. We selected 44 juvenile female rhesus macaques as subjects from a population of approximately 3000 macaques that inhabit a 475 -acre Sea Island. We obtained CSF and blood samples, and recorded behavioral observations over a subsequent 18-month period. Our results indicate an inverse correlation between CSF concentrations of the major serotonin metabolite 5-hydroxyindoleacetic acid (5-HIAA), and the frequency of low-intensity restrained aggression typically associated with matrilineal defense of social status. In contrast, previous research with males has shown an inverse correlation between CSF 5-HIAA concentrations and levels of violent unstrained aggression typically associated with traumatic injury and death. We also noted a negative correlation between plasma concentrations of the stress hormone cortisol and the frequency of low-intensity aggressive acts, a finding not reported in our previous studies with males. Further examination revealed a negative correlation between CSF 5-HIAA concentrations and the rate of long dangerous leaps through the forest canopy, suggesting that the relation between low serotonergic functioning and impulsivity may generalize to both female and male primates. These results indicate that females with low CSF 5-HIAA concentrations, like their male counterparts, are at increased risk for impulsive temperament, but that unlike males, females may be buffered from this risk through intersexual differences in life history patterns and social affiliation. Neuropsychopharmacology (2003) 28, I045-1055, advance online publication, 2 April 2003; doi: I 0. I038/sj.npp. I 300 I 7 I
\end{abstract}

Keywords: serotonin; 5-HIAA; aggression; Macaca mulatta; females; impulsivity

\section{INTRODUCTION}

Following a comprehensive review of the animal literature which consisted primarily of studies utilizing rodents, Soubrié (1986) concluded that deficits in central serotonin (5-HT), whether naturally occurring or pharmacologically induced, lead to deficits in waiting for reinforcement and withholding behavior to avoid punishment, and suggested that impaired CNS serotonin might form the basis of a number of psychopathological syndromes. Numerous studies have shown that men with psychopathological syndromes associated with impaired impulse control, such as explosive aggression and impulsive violence, and social incompetence exhibit low CNS serotonin 5-HT activity, as reflected in low cerebrospinal fluid (CSF) concentrations of

\footnotetext{
* Correspondence: Dr GC Westergaard, Division of Research, LABS of Virginia, Inc., 95 Castle Hall Road, PO Box 557, Yemassee, SC 29945, USA, Tel: + 843589 5190ext. 22, Fax: + 843589 5290,

E-mail: gwprimate@netscape.net

Received 25 October 2002; revised 06 January 2003; accepted 14 January 2003

Online publication: 22 January 2003 at http://www.acnp.org/citations/ Npp0I2203466
}

the major 5-HT metabolite 5-hydroxyindoleacetic acid (5HIAA) (Brown et al, 1979, 1982; Linnoila et al, 1983; Kruesi et al, 1990; Virkkunen et al, 1994a, b). Included among these deleterious patterns are high rates of criminality, alcohol dependence and abuse, and premature mortality from murder and suicide (Ballenger et al, 1979; Banki et al, 1981; Borg et al, 1985; Moss, 1987; Roy et al, 1989; Virkkunen et al, 1989). Although relations among serotonin, impaired impulse control, severe aggression, and social competence are less well-documented in women than in men, the limited literature base suggests a pattern of impulsive deficits that is consistent across sexes (Linnoila and Virkkunen, 1992; but see Manuck et al, 1998). Few studies have focused longitudinally on the role of serotonin in the development of impulse-deficit psychopathology in women, despite their frequent rates of suicide, child abuse, and alcohol and drug abuse (eg Fleming et al, 1998; Kelley, 1998; Hiroeh et al, 2001; Preuss et al, 2002).

Prospective studies of male rhesus macaques (Macaca mulatta), both in free-ranging and laboratory environments, have shown that animals with low concentrations of CSF 5-HIAA develop pathological patterns of antisocial behavior that parallel those seen in their human male 
counterparts. Included among these impulsive lifestyle patterns are severe unrestrained aggression, impulsive risk-taking, excessive alcohol intake, and violence leading to trauma and early mortality (Higley et al, 1992a, b, 1994, 1996b, c, 1997; Mehlman et al, 1994, 1995, 1997; Doudet et al, 1995; Kyes et al, 1995; Kalin, 1999). It is important to note that male macaques with low CSF 5-HIAA concentrations do not necessarily show higher rates of overall aggression than do males with high CSF 5-HIAA concentrations, but instead these monkeys show higher rates of only the most violent and severe forms of aggression that escalate to dangerous levels, such as prolonged chases and physical assaults (Higley et al, 1992b, 1996b, c, 1994, 1997; Mehlman et al, 1994, 1995, 1997). Interestingly, reviews of aggression in nonhuman primates generally indicate that overall rates of aggression are similar between the sexes (Higley, in press). However, because male macaques are larger and possess longer canines than female macaques, they are more likely than females to produce trauma and wounding through aggression. Within the laboratory, females with low CSF 5-HIAA concentrations are more likely than males to be removed from their groups as a result of severe wounding requiring veterinary treatment (Higley et al, 1996a). Our observations indicate that female violence produces tail and hip wounds in other females, and we have observed a number of cases where females produce severe trauma and at times even death using violent methods (Higley, personal observations). Little is known, however, with respect to direct female participation in impulsive aggression. No study has investigated the underlying psychobiology of female aggression in natural settings. Such a study is of critical importance in developing an animal model of violent behavior to provide evidence for its usage, underlying epidemiology, as well as the underlying biological variables that mediate such pathological behavior.

Studies of nonhuman primates have shown that patterns of aggression and violence in monkeys with low CSF 5HIAA concentrations are only one aspect of an overall psychopathological temperamental pattern of behavior. For example, nonhuman primate males with low CSF 5-HIAA concentrations tend to be socially isolated as evidenced by the reduced time spent in social interactions and increased distance from conspecifics (Higley et al, 1992b, 1996b, c; Mehlman et al, 1994, 1995, 1997). As in humans, much less is known concerning the relation between low CSF 5-HIAA concentration and patterns of affiliative behavior in female primates, and even less is known about the psychobiology of aggression in free-ranging animals. While in both humans and nonhuman primates violence is less likely in females than in males, laboratory studies suggest that the negative correlation between 5-HT turnover and aggression, and the positive correlation between 5-HT turnover and sociality, may generalize to both sexes (Higley et al, 1996a, d) — also see (Shively et al, 1995).

Despite evidence indicating that females with low CSF 5HIAA concentrations may show aggressive behaviors that parallel those in males with low CSF 5-HIAA concentrations, there is reason to believe that females may be buffered from the aggressive effects of impaired CNS serotonin functioning. This may be due in part to sex-based differences in life-history patterns. Perhaps, the most striking long-term difference between the sexes is that while males leave their natal groups to emigrate at or near the age of sexual maturity, females are closely bonded, and remain within their natal troops with their mothers and sisters as integral parts of smaller matrilineal-headed, extended families (Koford, 1965; Boelkins and Wilson, 1972; de Waal, 1989). As a result, gender differences oriented at maintaining close family relations are apparent at an early age. For example, some studies suggest that infant female rhesus macaques spend more time on their mother's ventrum than do infant males (Mitchell, 1968; Bappenheim and Tartabini, 1983). Further, as juveniles, females are less likely to engage in aggressive, rough, and tumble play than are males (e.g. Mason, 1960; Harlow, 1965), and instead spend more time in close proximity to their mothers and female kin in grooming bouts, a behavior widely seen as the 'glue' of sociality among macaques. Additionally, females are more likely to engage in infant care and interactions than are their male counterparts (Spencer-Booth, 1968).

In the wild, troops of rhesus macaques consist of 40-200 individuals divided into matrilineal-headed extended families with a grandmother, daughters, and their offspring. Matrilines are stratified by high-low social dominance rank, which is maintained by lifetime vigorous cooperative family defense, in which female kin typically defend one another as well as the offspring of both sexes (Lindburg, 1971; Berman, 1980, 1983; Bernstein and Ehardt, 1986; de Waal, 1989). High-ranking juveniles learn their social status early in life, and as a result of their kin's social support, often displace low-ranking adults during competitive challenges (Berman, 1980; de Waal, 1989). While males lose access to such social support during adolescence as they move to other troops, females maintain this family-oriented social support network throughout their lifetimes.

Given their close family support system and its quick kin response to threat, a buffering of the impulsive temperamental lifestyle in females would not be surprising. For example, females, who in contrast to males have the benefit of reliable support networks during competitive challenges, may be more likely to initiate and engage in aggressive interchanges. Moreover, such social support for females may buffer the deleterious effects of impulsivity, with other female members quickly coming to the aid of a related daughter, thereby reducing the probability of aggressive injury and social isolation.

To test this general theory of social buffering in females with low CSF 5-HIAA concentrations, in the present study we examined relations between CSF 5-HIAA and aggression using free-ranging female rhesus macaques. We also chose to study females to further develop our nonhuman primate model of violent, antisocial-like behavior, and specifically to assess whether the pathological problems seen in males with low CSF 5-HIAA concentrations differ or generalize across sexes. To investigate longitudinally the etiology of adult psychopathology, we began our investigation as early in development as possible under field conditions, looking at young females shortly after the birth of their younger siblings. Initiating our study at this age allowed us to begin early in life to assess subjects when they are just fully weaned and independent of the mother, and to look at how early interindividual differences predict future life patterns. 
We sought to produce as much as possible a homogeneous sample by selecting animals that are representative of the typical rhesus extended family. Since orphans and firstborns exhibit behavior patterns that are atypical when compared to other offspring (Hasegawa and Hiraiwa, 1980), and to provide a homogeneous sample of offspring to investigate and a sample that was most likely to generalize to the larger population, we selected females from families that represent the average female rhesus macaque, with a sibling, a mother who was still alive, and older sisters. We measured interindividual behavior characteristics of impulsivity and aggression that replicated those measured in previous publications of males with varying levels of CSF 5-HIAA concentrations and HPA functioning.

We tested the following two hypotheses: First, like males with low CSF 5-HIAA, female rhesus macaques with low CSF 5-HIAA concentrations would exhibit impaired impulse control, as measured by unprovoked leaps between trees at dangerous heights. Second, like males, females would exhibit an inverse relation between interindividual differences in CSF 5-HIAA concentrations and rates of severe and impulsive aggression, although we theorized that the close family system would attenuate this effect. As we have done with the males, we also assessed relations among monoamine metabolites, HPA functioning, and social affiliation.

\section{METHODS}

\section{Subjects and Design}

The subjects were 44 female rhesus macaques aged 12-18 months at the onset of this investigation. We determined subjects' ages using direct observations of births as recorded in the census data taken from field observations that were collected by a team of between four and eight trained observers. The animals are part of a free-ranging colony of approximately 3000 monkeys that reside on a 475acre South Carolina coastal Sea Island (Taub and Mehlman, 1989). At the time of sampling, the population was organized into 35 social groups with an adult sex ratio of 2.5 females to one male. An exhaustive list of all available subjects with a living mother, new sibling, and an older sister in the pre-established age range was generated and subjects were sequentially selected from that list as they were captured. While our trapping procedure is passive virtually all yearlings are trapped during the trapping season, making a biased sampling unlikely.

\section{Physiological Sampling}

We obtained physiological data from 44 subjects over two sampling periods, separated by 2 months, as the animals were trapped in baited corrals (diameter of each corral $=10-25 \mathrm{~m}$, height of corral walls $=4 \mathrm{~m})$. Subjects were marked with a commercial dye for easy identification and were released back into their free-ranging environment. CSF and blood samples were obtained from each monkey at each of the captures. During the first sampling period physiological samples were obtained from 39 monkeys. At the time of the second sampling period, 27 of the original study subjects were captured, and five additional subjects were added to the study. Owing to technical difficulties, CSF samples could not be obtained from two subjects during the second sampling period.

To capture the monkeys, on the first day of each 5-day trapping period, the aluminum corrals where the monkeys receive their daily rations were baited with food and the doors were closed. Monkeys voluntarily jumped from the top of the walls down into the corrals to obtain the food or to be with the other captured animals, but were unable to escape because they could not jump out over the high corral walls. Following trapping, the monkeys were hand captured using large nets, anesthetized using ketamine hydrochloride $(10 \mathrm{mg} / \mathrm{kg})$, and weighed on a commercial scale accurate to within $0.25 \mathrm{~kg}$. CSF and blood samples were drawn within $30 \mathrm{~min}$ of entering the capture corral. A volume of $2 \mathrm{ml}$ of CSF was obtained from the cisterna magna of each subject using a 22-gauge needle and a $5 \mathrm{~cm}^{3}$ syringe. The samples were then immediately aliquoted, quick frozen, and maintained on dry ice. Blood samples were obtained by venipuncture, then centrifuged using a portable centrifuge, and placed in dry ice. The blood was then transported to a laboratory on the mainland, where it was stored with the $\mathrm{CSF}$ at a temperature of $-70^{\circ} \mathrm{C}$. CSF samples were assayed for 5-HIAA, homovanillic acid (HVA), and 3-methoxy-4hydroxyphenylglycol (MHPG) using high-performance liquid chromatography with electrical detection as described by Scheinin et al (1983). All intra- and interassay coefficients were less that $10 \%$. Plasma samples were assayed for cortisol, adrenocorticotrophin (ACTH), and prolactin using radioimmunoassays (Abraham et al, 1972). To control for how long each monkey was in the trap before being restrained for sampling, all of the females in the 10 corrals were captured each day, and released following sampling. Thus, no subject spent more than 1 day in a capture corral. To further control for this and other possible procedural confounds that might influence CSF and plasma values, records were kept of the elapsed minutes from when the technicians initially entered the corral until the CSF or blood sample was obtained; time of day and day of the week were also recorded for each sample. Weight of the subject animal was recorded within $0.1 \mathrm{~kg}$. Following physiological sampling subjects were released with other members of their group back into the area of their home range.

\section{Behavioral Sampling}

Of the 44 subjects from whom we collected physiological samples, we were able to collect behavioral samples from 41 . Subjects were located in the field by trained observers familiar with the daily movements of each troop. These technicians observed focal animals directly or through binoculars depending on the distance of the animal from the observer. Two observers collected four 30-min sessions of behavioral data per subject over an 18-month period. The samples were distributed such that two behavioral data collection sessions were conducted when the animals were 1 year old, and two sessions were conducted when the animals were 2 years old. Of the original 44 females from which we obtained physiological samples, two died during the early part of the study and one could not be found during the behavioral sampling and was presumed dead. The observers used a focal animal sampling method and 
randomized data collection sessions for subject order and time of day. Social behavior categories followed those used in our studies of male rhesus monkeys described in detail elsewhere (Mehlman et al, 1994). The observers recorded the following behaviors: (1) proximity; (2) social grooming; (3) submission; (4) leaping between trees at dangerous heights; and (5) aggression. Aggressive behaviors were defined and categorized by intensity into displacements, stationary threats, chases, and physical assaults. These behaviors were treated as frequency data for which we calculated rates per hour. For the purpose of analysis, the data were summarized into moderate (displacements and threats) and severe (chases and assaults) aggression. The scoring system distinguished between the animal that initiated grooming or aggression from the animal that received grooming or aggression. Leaping, aggression, submission, and social grooming were scored independent of play.

Family dominance rankings were determined by assessing the directionality of dyadic outcomes between adult females of the same troop. A preferred food item (corn) was thrown between two females, and a 'winner' and 'loser' were scored when one female clearly submitted to the other by fear grimacing, avoiding, fleeing, etc. and the remaining female took the food item. Only clear submissions were scored. We determined rank by tabulating all group females in a matrix using the outcomes of these dyadic interactions, and assigned each adult female in a group a numerical rank. In order to control for group size and differing numbers of matrilines between social groups, we generated rank indices by dividing the numerical rank by the total number of individuals in the group. Offspring, including the females in this study, were assigned the rank of their matriline.

Unprovoked leaping behavior was scored as frequency data, falling into one of the three categories of increasing danger: (1) short leaps, (2) medium leaps, and (3) long leaps. Only those leaps occurring at a height of $7 \mathrm{~m}$ or greater were scored (judged to be high enough to be potentially harmful if a fall occurred). Leaps were only scored if they were unprovoked, and not part of a social or aggressive sequence. Leaps estimated to traverse less than $1 \mathrm{~m}$ of open space were scored as short. Leaps of $1 \mathrm{~m}$ to less than $3 \mathrm{~m}$ were scored as medium, and leaps greater than $3 \mathrm{~m}$ were scored as long. Since the overall rate of activity could influence the rate of leaping and thus the raw frequency of short, medium, and long leaps, a derived variable was calculated indicating the percentage of total leaps that were short, medium, and long. 'Long leap ratio', for example, was calculated by dividing the total number of long leaps by the total number of all leaps; it therefore represents the proportion of overall leaping that could be considered the most dangerous to the subject. Each occurrence of other behaviors was treated as an event with a corresponding duration.

\section{Data Analysis}

The descriptive statistics for each variable are presented in Table 1. Paired $t$-tests were used to test for differences in CSF monoamine and plasma cortisol concentrations across sampling times 1 and 2 . Univariate linear analyses were conducted by correlation $z$-tests and regression to test for correlations between physiological measures, behavioral variables, and possible confounding factors (see Tables 2 and 3). To assure that our analyses were not a product of extremes and to control for extraneous variables that may have affected the subject's physiology, individuals with values two or more standard deviations above or below the mean were excluded from our analyses. Since cortisol values were correlated with the time of day in which samples were taken during the first sampling period (see Table 2), multiple regression analysis controlling for this factor was used when correlating cortisol with any behavioral measure. Additionally, CSF HVA, CSF 5-HIAA, and CSF MHPG concentrations were significantly positively correlated; thus, to assess the independent contribution of these variables

Table I Descriptive Statistics for Physiological and Behavioral Variables

\begin{tabular}{|c|c|c|c|c|c|c|c|c|}
\hline & $\begin{array}{c}\text { Age } \\
\text { (months) }\end{array}$ & $\begin{array}{c}\text { Weight } \\
\text { (kg) }\end{array}$ & $\begin{array}{l}\text { CSF 5-HIAA } \\
(\mathrm{pm} / \mathrm{ml})\end{array}$ & $\begin{array}{c}\text { CSF HVA } \\
(\mathrm{pm} / \mathrm{ml})\end{array}$ & $\begin{array}{c}\text { CSF MHPG } \\
(\mathrm{pm} / \mathrm{ml})\end{array}$ & $\begin{array}{c}\text { Plasma } \\
\text { cortisol } \\
(\mu \mathrm{g} / \mathrm{dl})\end{array}$ & $\begin{array}{c}\text { Plasma } \\
\text { ACTH } \\
(\mathrm{pg} / \mathrm{ml})\end{array}$ & $\begin{array}{c}\text { Plasma } \\
\text { prolactin } \\
\text { (ng/ml) }\end{array}$ \\
\hline \multicolumn{9}{|c|}{ Biological variables } \\
\hline Mean & 21.8 & 2.9 & 414.6 & 3372.7 & 271.7 & 63.1 & 289.0 & 3.6 \\
\hline SD & 1.8 & 0.4 & | 46.1 & 697.7 & 58.5 & 15.8 & 201.6 & 5.0 \\
\hline$n$ & 44 & 43 & 44 & 44 & 44 & 44 & 44 & 44 \\
\hline \multicolumn{9}{|c|}{ Leaping } \\
\hline & $\begin{array}{l}\text { Short } \\
\text { leaps }\end{array}$ & $\begin{array}{l}\text { Medium } \\
\text { leaps }\end{array}$ & $\begin{array}{l}\text { Long } \\
\text { leaps }\end{array}$ & $\begin{array}{l}\text { Total } \\
\text { leaps }\end{array}$ & $\begin{array}{l}\% \text { Long } \\
\text { leap }\end{array}$ & & & \\
\hline Mean & 16.10 & 6.60 & 0.35 & 39.80 & 0.02 & & & \\
\hline SD & 9.80 & 4.80 & 0.45 & 28.70 & 0.02 & & & \\
\hline$n$ & 41 & 41 & 41 & 41 & 41 & & & \\
\hline \multicolumn{9}{|c|}{ Agonism } \\
\hline & $\begin{array}{c}\text { Overall } \\
\text { aggression }\end{array}$ & $\begin{array}{l}\text { High-intensity } \\
\text { aggression }\end{array}$ & $\begin{array}{c}\text { Low-intensity } \\
\text { aggression }\end{array}$ & $\begin{array}{l}\text { Received } \\
\text { aggression }\end{array}$ & & & & \\
\hline Mean & 2.1 & 0.7 & 1.5 & 4.6 & & & & \\
\hline SD & 2.1 & 0.8 & 1.8 & 3.7 & & & & \\
\hline$n$ & 41 & 41 & 41 & 41 & & & & \\
\hline
\end{tabular}

All behaviors are reported as mean number of acts per hour of observation. 
Table 2 Relationships of Possible Confounding Variables to Physiological Values/Time I

\begin{tabular}{lcrrrrr}
\hline Confounding variable & $\begin{array}{c}\text { 5-HIAA } \\
\mathbf{n}=\mathbf{3 8} \\
\text { Correlation }\end{array}$ & $\begin{array}{c}\text { HVA } \\
\mathbf{n}=\mathbf{3 8}\end{array}$ & $\begin{array}{c}\text { MHPG } \\
\mathbf{n = 3 8}\end{array}$ & $\begin{array}{c}\text { Cortisol } \\
\mathbf{n = 3 6}\end{array}$ & $\begin{array}{c}\text { ACTH } \\
\mathbf{n = 3 8}\end{array}$ & $\begin{array}{c}\text { Prolactin } \\
\mathbf{n}=\mathbf{3 8}\end{array}$ \\
\hline Day of the week & 0.02 & 0.08 & 0.27 & -0.20 & -0.27 & 0.01 \\
Time of day & 0.20 & 0.18 & 0.02 & $-0.34 *$ & 0.23 & 0.00 \\
Time to ketamine & -0.26 & -0.27 & -0.23 & 0.31 & 0.05 & -0.02 \\
Time to venipuncture & 0.15 & 0.20 & 0.30 & 0.00 & -0.10 & -0.15 \\
Time to CSF & 0.13 & 0.18 & 0.32 & 0.18 & -0.05 & -0.13 \\
Weight & -0.12 & -0.09 & -0.10 & -0.23 & -0.06 & -0.11 \\
\hline
\end{tabular}

*Significant at the $95 \%$ confidence level.

Table 3 Relationships of Possible Confounding Variables to Physiological Values/Time 2

\begin{tabular}{|c|c|c|c|c|c|c|}
\hline Confounding variable & $\begin{array}{c}\text { 5-HIAA } \\
n=30 \\
\text { Correlation }\end{array}$ & $\begin{array}{c}\text { HVA } \\
n=30\end{array}$ & $\begin{array}{c}\text { MHPG } \\
n=30\end{array}$ & $\begin{array}{c}\text { Cortisol } \\
n=30\end{array}$ & $\begin{array}{c}\text { АСТH } \\
n=30\end{array}$ & $\begin{array}{c}\text { Prolactin } \\
n=\mathbf{3 0}\end{array}$ \\
\hline Day of the week & -0.19 & -0.20 & -0.21 & -0.10 & -0.19 & 0.25 \\
\hline Time of day & 0.22 & 0.23 & -0.11 & -0.09 & 0.35 & 0.38 \\
\hline Time to ketamine & 0.30 & 0.02 & 0.08 & -0.11 & 0.13 & -0.28 \\
\hline Time to venipuncture & 0.01 & 0.02 & 0.13 & -0.21 & -0.30 & -0.21 \\
\hline Time to CSF & -0.28 & -0.24 & -0.15 & 0.04 & -0.08 & -0.04 \\
\hline Weight & -0.08 & -0.26 & -0.24 & -0.11 & -0.19 & 0.12 \\
\hline
\end{tabular}

* Significant at the 95\% confidence level.

when they were correlated with behaviors, multiple regression was used with the monoamine concentrations being entered as independent variables, and the behaviors being tested were the dependent variables.

\section{RESULTS}

\section{Correlations Among Physiological Measures}

Mean CSF monoamine metabolite and plasma hormone concentrations for the two samples (Time 1 and Time 2) did not differ between the two samples for CSF 5-HIAA, ACTH, and prolactin concentrations ( $p>0.05$ in all cases), but significant differences were found in concentrations between Time 1 and Time 2 for CSF HVA, CSF MHPG, and plasma cortisol (for CSF HVA: $t(24)=2.70, p<0.02$; for CSF MHPG: $t(24)=2.26, p<0.04$; for cortisol: $t(26)=2.73$, $p<0.02)$. The samples taken at Time 1 were significantly correlated with the samples taken at Time 2 (for CSF 5HIAA: $r(23)=0.47, p<0.02$; for CSF HVA: $r(23)=0.43$, $p<0.04$; for CSF MHPG: $(r(23)=0.48, p<0.02$; for cortisol: $r(25)=0.56, p<0.002$; for ACTH: $r(25)=0.38, p<0.05)$; for prolactin: $(r(25)=0.38, p=0.05)$. Given these significant positive correlations, we formulated a mean value for each biochemical in order to assess interindividual correlations between each biochemical we measured and behavior. Where mean values differed between the two samples (CSF HVA, CSF MHPG, and plasma cortisol) data were standardized using an $z$-score transformation, and mean $z$-scores were used in subsequent analyses. Average CSF HVA, CSF 5-HIAA, and CSF MHPG concentrations were significantly positively correlated (for CSF HVA and CSF 5-HIAA: $r(42)=0.61, p<0.0001$, for CSF HVA and CSF
MHPG: $r(42)=0.70, p<0.0001$; for CSF 5-HIAA and CSF MHPG: $r(42)=0.64, p<0.0001)$.

\section{Behaviors}

We examined family dominance rank as a predictor of low intensity, high intensity, and total aggression frequencies. We found that aggression frequency did not differ by dominance rank in the study sample. Additionally, rank was not a reliable predictor of affiliative social behaviors, which included time spent grooming, time spent receiving groom, and time spent in proximity to other animals. Rank was not correlated with measures of leaping or our measure of impulsivity, that is, long leap ratios ( $p>0.05$ in all cases).

Univariate, linear analysis indicated that the long leap ratio was positively correlated with both high-intensity and low-intensity aggression, with the correlation stronger for high-intensity aggression (high-intensity aggression: $r(39)=0.46, p<0.002$; low-intensity aggression: $r(39)=$ $0.35, p<0.03)$, and overall total aggression $(r(39)=0.42$, $p<0.006)$. The same ratios derived for both short and medium leaps were not significantly correlated with any measure of aggressive behavior. Neither leaping nor long leap ratio was significantly correlated with affiliative social behaviors, which included time spent grooming social partners, time spent receiving groom, or time spent in proximity to conspecifics.

\section{CSF Monoamine Metabolites and Aggression}

Univariate linear analysis indicated significant correlations between both CSF 5-HIAA concentrations and total aggression, and CSF HVA concentrations and total aggression (for CSF 5-HIAA: $r(39)=-0.34, p<0.03$; for CSF 
Table 4 CSF Monoamine Metabolites, Plasma Hormones and Aggression

\begin{tabular}{lcccccc}
\hline & $\begin{array}{c}\text { 5-HIAA } \\
\mathbf{n = 4 I} \\
\text { Correlation }\end{array}$ & $\begin{array}{c}\text { HVA } \\
\mathbf{n}=\mathbf{4 I}\end{array}$ & $\begin{array}{c}\text { MHPG } \\
\mathbf{n = 4 I}\end{array}$ & $\begin{array}{c}\text { Cortisol } \\
\mathbf{n}=\mathbf{3 9}\end{array}$ & $\begin{array}{c}\text { ACTH } \\
\mathbf{n = 3 9}\end{array}$ & $\begin{array}{c}\text { Prolactin } \\
\mathbf{n}=\mathbf{3 9}\end{array}$ \\
Behavior & -0.16 & -0.14 & -0.11 & -0.17 & -0.08 & 0.12 \\
\hline High-intensity aggression & $-0.34 *$ & $-0.40^{*}$ & -0.24 & $-0.35 *$ & 0.02 & 0.01 \\
Low-intensity aggression & $-0.34 *$ & $-0.39 *$ & -0.24 & $-0.34 *$ & 0.00 & 0.04 \\
Total aggression & 0.15 & 0.14 & 0.26 & 0.01 & -0.13 & 0.05 \\
Received aggression & & & & &
\end{tabular}

*Significant at the $95 \%$ confidence level.

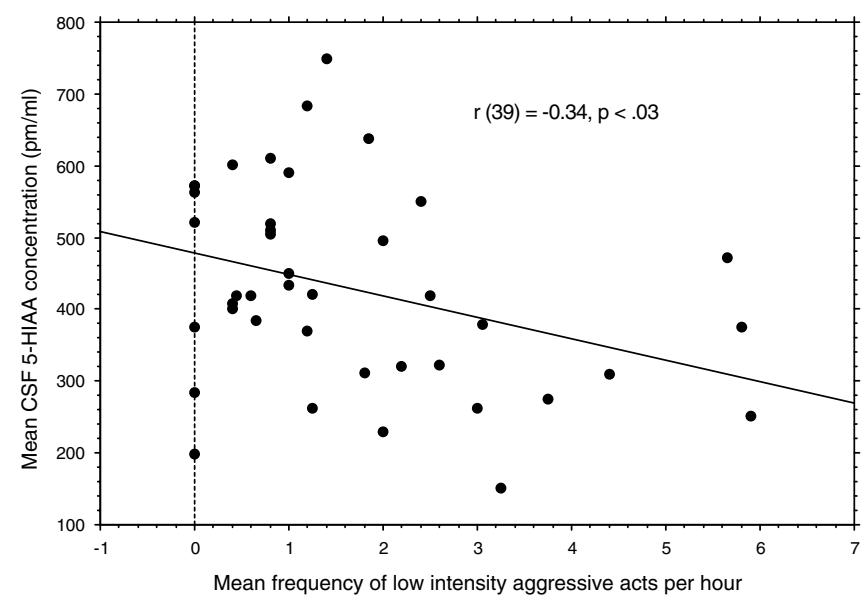

Figure I Scatterplot showing the relation between mean CSF 5-HIAA concentration $(\mathrm{pm} / \mathrm{ml})$ and the mean frequency of low-intensity aggressive acts directed towards other animals per hour of focal observation.

HVA: $r(39)=-0.39, p<0.02$, see Table 4$)$. Further analysis indicated that CSF 5-HIAA and CSF HVA concentrations were each negatively correlated with the frequency of lowlevel aggression, that is, those bouts of aggression that did not result in direct physical conflict, but not with highintensity aggression, that is, chases and contact aggression (for CSF 5-HIAA and low-intensity aggression: $r$ $(39)=-0.34, p<0.03$; for CSF HVA and low-intensity aggression: $r(39)=-0.40, p<0.01$, see Table 4, Figure 1). CSF MHPG concentrations were not significantly correlated with behavioral indices of aggression in this sample of females (see Table 4). When multiple regression analysis was used to assess the relation between low-intensity aggression while controlling for the positive correlation between CSF 5-HIAA and CSF HVA concentrations, the overall $R$ between the two monoamine metabolites and aggression remained significant $(R(2,38)=0.45, p<0.02)$; however, with the reduced degrees of freedom, the partial correlation for each metabolite failed to achieve statistical significance (CSF 5-HIAA $r=-0.18, p<0.28$; CSF HVA $r=0.33, p<0.07)$.

\section{Plasma Hormones}

Univariate linear analysis indicated a negative correlation between plasma cortisol and overall aggression (cortisol and total aggression: $r(37)=-0.34, p<0.04$, see Table 4 ).

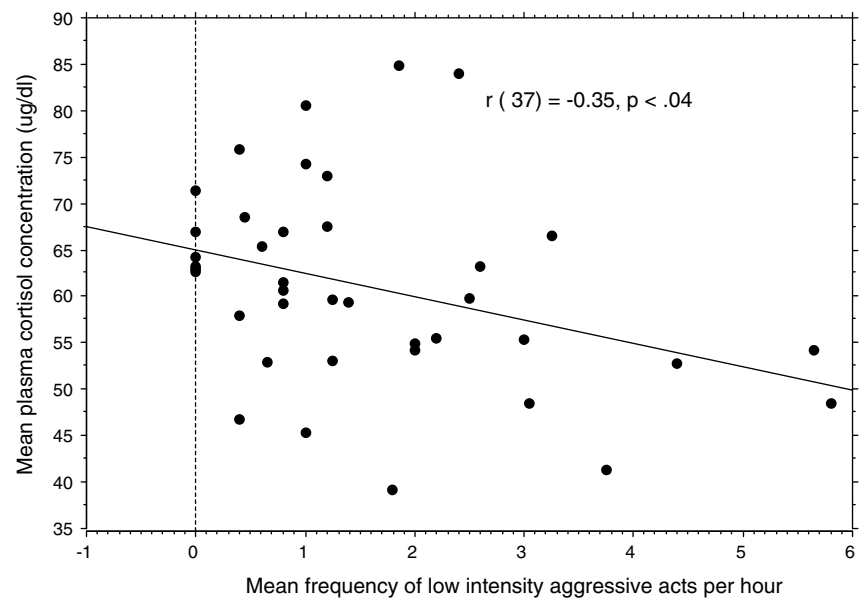

Figure 2 Scatterplot showing the relation between mean plasma cortisol concentration $(\mu \mathrm{g} / \mathrm{dl})$ and the mean frequency of low-intensity aggressive acts directed towards other animals per hour of focal observation.

Further analysis showed that this correlation was largely because of the correlation between low-intensity aggression and cortisol. Plasma cortisol concentrations were lower in subjects that engaged in high rates of low-intensity aggression (cortisol and low-intensity aggression: $r$ $(37)=-0.35, p<0.04$, see Figure 2), but there was no correlation between cortisol and frequent high-intensity aggression (i.e. severe bouts of aggression that involved chases and violent attacks). Plasma ACTH and prolactin concentrations were not significantly correlated with frequencies of total, low-intensity, or high-intensity aggressive acts.

\section{Physiological Correlates of Impulsivity}

Univariate linear analysis indicated a negative correlation between the percentage of long leaps and CSF 5-HIAA concentration $(r(39)=-0.32, p<0.05$, see Table 5 and Figure 3$)$. The same negative correlation was found with respect to the percentage of long leaps and both CSF MHPG $(r(39)=-0.34, p<0.04)$ and CSF HVA $(r(39)=-0.37$, $p<0.02)$. When multiple regression analysis was used to control for the positive correlation between CSF 5-HIAA and CSF HVA concentrations, with the reduced degrees of freedom, the overall $R$ was not significant $(R(3,37)=40$, $p<0.10$ ), and none of the metabolites approached a level of statistical significance $(r>0.25)$. Univariate linear analysis 
Table 5 CSF Monoamine Metabolites, Plasma Hormones, and Leaping

\begin{tabular}{lcccrrr}
\hline & $\begin{array}{c}\text { 5-HIAA } \\
\mathbf{n = 4 I} \\
\text { Leap type }\end{array}$ & $\begin{array}{c}\text { HVA } \\
\mathbf{n = 4 I}\end{array}$ & $\begin{array}{c}\text { MHPG } \\
\mathbf{n = 4 I}\end{array}$ & $\begin{array}{c}\text { Cortisol } \\
\mathbf{n = 3 9}\end{array}$ & $\begin{array}{r}\text { ACTH } \\
\mathbf{n = 4 I}\end{array}$ & $\begin{array}{r}\text { Prolactin } \\
\mathbf{n = 4 I}\end{array}$ \\
\hline Short leap ratio & -0.04 & 0.17 & 0.10 & -0.18 & -0.07 & 0.13 \\
Medium leap ratio & 0.11 & -0.09 & -0.02 & 0.15 & 0.10 & -0.13 \\
Long leap ratio & $-0.32 *$ & $-0.37 *$ & $-0.34 *$ & 0.16 & -0.02 & -0.03 \\
Total leaps & 0.13 & -0.19 & -0.12 & -0.09 & 0.03 & -0.13 \\
\hline
\end{tabular}

* Significant at the 95\% confidence level.

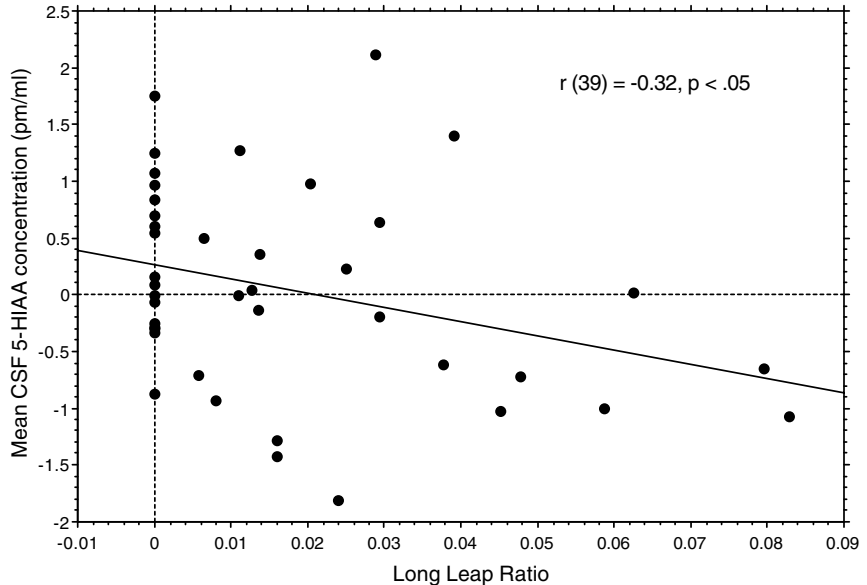

Figure 3 Scatterplot showing the relation between mean CSF 5-HIAA concentration $(\mathrm{pm} / \mathrm{ml})$ and our measure of impulsivity, long leap ratio (number of long leaps/total number of leaps).

revealed that none of the plasma hormone concentrations were associated with the long leap ratio. No significant correlations were found for the percentages of other types of leaps (short or medium distances) and monoamine metabolite or plasma hormone concentrations.

\section{DISCUSSION}

The positive interindividual correlation between the two CSF 5-HIAA samples is one of our most replicated findings, and in this study we show for the first time that it is present among free-ranging females. Our studies and others that have repeatedly sampled CSF from the same individuals have shown that in the controlled laboratory environment interindividual differences in CSF 5-HIAA concentrations remain stable over time and across situations in both males and females (Higley, in press). Given the varied life events and situations that free-ranging females experience between samplings (fights, access to food, changing maternal support, etc.), our findings suggest that interindividual stability in the serotonin system is most likely a function of a trait-like response of the CNS system. Such findings suggest that as a potential risk factor, trait-like impaired CNS serotonin functioning may underlie a risk for violent aggression and other impulse control deficits.

The significant positive correlation between CSF 5-HIAA and HVA concentrations found here has also been found in young male macaques (Higley et al, 1992b; Mehlman et al, 1994, 2000). However, in contrast to our previous findings showing a negative correlation between CSF MHPG and HVA concentrations in males (Mehlman et al, 2000), the results of the present study indicate positive correlations between CSF MHPG, and both CSF 5-HIAA and HVA concentrations. The hereditary and environmental factors influencing the strong correlations among monoamine metabolite concentrations, and sex-based differences among these, are beyond the scope of the present study. However, it is interesting to note that preliminary results from studies in humans indicate sex-based differences in the expression of a putative functional promoter polymorphism in the monoamine oxidase A gene (Jonsson et al, 2000). Specifically, among women carrying the allele associated with more efficient transcription, CSF 5-HIAA and HVA concentrations were higher than in women lacking the allele, while the opposite trend was true for men. We are currently investigating ways to evaluate this in our subject sample, in order to elucidate the genetic bases of monoamine and monoamine oxidase turnover in nonhuman primates, as well as the implications of these for social behavior.

Our data suggest that females with low CSF 5-HIAA concentrations are more impulsive than are females with high CSF 5-HIAA concentrations. In the present study, we found that CSF 5-HIAA concentrations were negatively correlated with the proportion of spontaneous, unprovoked long leaps, but not with overall leaping frequency, suggesting that the negative correlation between dangerous leaping and CSF 5-HIAA concentration is not simply a result of higher activity in females with low CSF 5-HIAA concentrations. The results of the present study suggest that the negative correlation between impaired serotonin function and impulsive behavior found in males may generalize to females among M. mulatta. However, unlike in our research with males, we found that CSF HVA and CSF MHPG were correlated with impulsive leaping in our female subject sample. Age differences cannot be ruled out as contributing to this difference between sexes, because we first recorded impulsive leaping in our male subjects at a somewhat later age than in our female subjects. Alternatively, one speculative but related explanation for the relation between the dopamine system and dangerous, spontaneous long leaps is that at this younger age, sensation seeking may play a role in this behavior. Both the dopamine and noradrenergic systems have been linked to sensation seeking in humans (Zuckerman, 1985). Moreover, low anxiety is linked to low levels of CSF MHPG in some studies (Redmond et al, 
1986). It seems likely that anxious animals would be less likely to venture into situations of risk. Thus, it may be that in females, impulsivity, sensation seeking, and low anxiety underlie this dangerous behavior. Further testing of this hypothesis will require replication in older female subjects.

Replicating in part the results from studies with male primates (Higley et al, 1992b, 1994, 1996c, 1997; Mehlman et al, 1994, 1995, 1997), there was a negative correlation between agonistic behavior and CSF 5-HIAA concentrations. However, unlike findings in our male subjects, the more severe and dangerous high-intensity aggression was not significantly correlated with CSF concentrations of 5HIAA. Lower intensity, restrained aggression that is typically used to defend status, on the other hand, was negatively correlated with CNS serotonin turnover. Interestingly, long leaping, our measure of impulsivity, was correlated with low intensity and overall aggression. These findings indicate that among females, at least those living in natural settings, low-intensity aggression is correlated with low CSF 5-HIAA concentrations, and that low CNS serotonin turnover and impulsivity may in part underlie aggressive behavior in females.

There are gender differences in the expression of aggression in male and female macaques that may explain in part the difference between the males who showed a strong correlation between escalated aggression and CSF 5HIAA concentrations, and the females who showed no relation between high-intensity aggression and CSF 5-HIAA concentrations. Male and female rhesus macaques engage in relatively equal amounts of overall aggression in natural environments; however, females are less likely to engage in severe aggression, and are less likely to be severely wounded, than are males (Lindburg, 1971). Further, females tend to receive their wounds in the hind area, presumably while avoiding aggression, whereas males are more likely to engage in frontal assaults that result in wounds to the face and frontal area (Hausfater, 1972). Studies show that within groups of macaques, female kin spend more time in close proximity than do males (Berman, 1983) and perhaps as a result, females are more likely to defend their female offspring and kin than their male relatives (Bernstein and Ehardt, 1986). That females spend more time in the presence of close kin may also explain why among females the target of aggression is often a family member. Such aggression seldom escalates to severe levels.

While we were not able to ascertain with whom our subjects were fighting, one possible related explanation is that in the context of family and close kin, aggression in juvenile females with low CSF 5-HIAA concentrations is more likely to be directed at relatives, and perhaps as a consequence, aggression is expressed at a lower level than is seen in the males who spend more of their time with nonkin. We are in the process of placing identifying marks on the close kin of our subjects in order to test this hypothesis. Consistent with this interpretation, impulsive long leaps were also correlated with low-intensity aggression, suggesting an impulsivity link to low-intensity aggression, a finding that is unlike our data from males.

As we have seen in male rhesus macaques, high-intensity aggression was correlated with impulsive long leaps in our female subjects, suggesting that impulse control deficits are an aspect of violent aggression among females as well as males. In related research, Higley et al (1996a) found that in the laboratory, when unfamiliar female rhesus macaques were placed together to form a social group, wounds were more frequently observed among subjects who possessed low CSF 5-HIAA concentrations than among subjects with high CSF 5-HIAA concentrations. In another study of unrelated laboratory-dwelling females who had been placed together to form a breeding group, subjects with low CSF 5HIAA concentrations engaged in relatively high rates of severe aggression (Westergaard et al, 1999), suggesting that high-intensity aggression of a violent nature is a characteristic of females, as well as males, with low CSF 5-HIAA concentrations. In a previous study (Westergaard et al, 1999), we found a parallel species difference, with rhesus macaques, who are reportedly one of the more aggressive macaque species (Bernstein and Gordon, 1974), exhibiting lower CSF 5-HIAA concentrations, higher rates of serious and escalated aggression, and a higher incidence of wounding, when compared to the more placid and friendly pigtailed macaques (Macaca nemestrina). Additionally, in females of a closely related species (Macaca fascicularis), Shively et al (1995) found that rates of aggression were negatively correlated with the prolactin response to a fenfluramine challenge, although it must be noted that the authors did not differentiate impulsive aggression from general aggression.

In all of these studies, severe aggression was observed between unrelated females. In contrast, females in the present study lived in matrilineal families. We found that low serotonergic turnover was correlated with high frequencies of overall aggression, and specifically with low-intensity aggression. Given that this is the first study of the relation between aggression and serotonin in females living in the natural setting, it is plausible that the reason for the discrepant finding between previous studies of laboratory dwelling rhesus females and the current study may be because of factors intrinsic to the natural environment. We suggest that this may be explained in terms of differences between the genders in the natural life history and close family bond among this female-resident species. Given the strong female social bonds and social support that characterizes the rhesus macaque social system, the consequences of low serotonergic turnover may be buffered by the family and kin in agonistic situations.

As with the serotonin system, CSF concentration of the dopamine metabolite HVA was correlated with lowintensity aggression but not with high-intensity aggression. In studies with males, multiple regression analysis revealed that when the common correlation between CSF 5-HIAA and CSF HVA was controlled, only the independent contribution of CSF 5-HIAA remained significant (Mehlman et al, 1995; Higley et al, 1996b). In the current study, when multiple regression was performed, both systems remained as contributors to aggression. This is not consistent with laboratory studies of older females, where only CSF 5-HIAA concentrations were correlated with aggression (Higley et al, 1996a). Subsequent studies will be needed to further understand the relative contributions of the serotonin and dopamine systems in the expression of impulsivity and aggression in females. 
Give and receive groom, and social proximity, were all correlated and closely intertwined, suggesting a constellation of social behaviors indigenous to an individual subject. Sociality has been shown to be trait like in rhesus macaques, enduring over time and situation (eg see Higley and Suomi, 1989). Affiliative behaviors did not correlate with any of the physiological measures, a finding consistent with our early research in juvenile males (Mehlman et al, 1995). In studies with males, positive correlations emerged between serotonin functioning and sociality only after the males were older and more independent of their mothers. We are following these female subjects to assess whether such relationships are found in older subjects and whether these behavior and physiological measures are related to rank in the natural setting.

Surprisingly, high levels of plasma cortisol were correlated with low rates of overall and low-intensity aggression, but not with high-intensity aggression. Aggression carries with it both a risk of physical injury and a risk of social alienation. It is reasonable to expect that cortisol would be higher in the females that engaged in the most aggression, since presumably they are under more stress. Moreover, in this study receiving aggression was not correlated with cortisol. Also, at variance with these findings are the results from our original study of male aggression (Higley et al, $1992 b)$. In that study, subjective rankings for aggression, based chiefly on the frequency and location of scars and wounds, were positively correlated with plasma cortisol and ACTH concentrations. High-ranking animals typically engage in more frequent low-level aggression to defend their status and some studies show that high-ranking animals have higher cortisol levels. While it seems reasonable to interpret this in terms of a social dominance model in which high-ranking animals engage in a high rate of lowintensity aggression to defend status (Higley and Suomi, 1996), none of the social dominance measures were correlated with aggression. This may be a result of social buffering of higher-ranking mothers to whom the juvenile females were still partially dependent. While the underlying reason cannot be ascertained, our data suggest that the females that initiate and engage in high rates of low-level aggression were under less stress than those that seldom initiate aggression. One difficulty in interpreting this finding is that with our current system it is not possible to ascertain the identity of the target of the subjects' aggression. It is also important to note that the present study addresses questions of aggression only among juvenile females. Since aggression may serve different functions depending on the age, sex, and relatedness of the target (eg maintaining status, obtaining food from an infant, or taking part in a large coalition), we have modified our scoring system to allow us to identify targets of aggression. We continue to collect physiological and behavioral data for our subject sample of females in order to examine the relations among monoamine and plasma hormone concentrations and behavior during adulthood.

In summary, we found that CSF 5-HIAA concentrations correspond negatively with rates of low-level aggression as well as with the proportion of long dangerous leaps in this sample of free-ranging rhesus macaque females. These results support the view that CNS serotonin and possibly dopamine functioning is related to agonism and impulsive control in female primates. In this species, the effects of low serotonergic turnover may be buffered in agonistic situations as a result of strong social support among females, such that low-level aggression associated with defense of status, rather than high-level violent aggression, is associated with low CSF 5-HIAA concentrations.

\section{ACKNOWLEDGMENTS}

We acknowledge the contributions of Beth Hoos and Patrick Mehlman during the design and early data collection phases of this study. This research was conducted with support from FDA Contract 223-01-1101 and NIAAA Contract N01AA02018. The LABS of Virginia, Inc. Institutional Animal Care and Use Committee approved a research protocol for this study in accordance with and as required by the Animal Welfare Act.

\section{REFERENCES}

Abraham GE, Buster JE, Teller RC (1972). Radioimmunoassay of plasma cortisol. Anal Lett 5: 757-766.

Ballenger JC, Goodwin FK, Major LF, Brown GL (1979). Alcohol and central serotonin metabolism in man. Arch Gen Psychiatry 36: 224-227.

Banki CM, Molnar G, Fekete I (1981). Correlation of individual symptoms and other clinical variables with cerebrospinal fluid amine metabolites and tryptophan in depression. Arch Psychiatr Nervenkr 229: 345-353.

Bappenheim J, Tartabini A (1983). Different behavior patterns of mother-infant interactions. Acta Anthropogenet 7: 13-25.

Berman CM (1980). Early agonistic experience and rank acquisition among free-ranging infant rhesus monkeys. Int J Primatol 1: 153-170.

Berman CM (1983). Influence of close female relatives on peerpeer rank acquisition. In: Hinde RA (ed). Primate Social Relationships: An Integrated Approach. Sinauer Associates Inc.: Sunderland, MA. pp 157-159.

Bernstein IS, Ehardt CL (1986). Selective interference in rhesus monkey (Macaca mulatta) intragroup agonistic episodes by age-sex class. J Comp Psychol 100: 380-384.

Bernstein IS, Gordon TP (1974). The function of aggression in primate societies. Am Sci 62: 304-311.

Boelkins RC, Wilson AP (1972). Intergroup social dynamics of the Cayo Santiago rhesus with special reference to changes in group membership by males. Primates 13: 125-140.

Borg S, Kvande H, Liljeberg P, Mossberg D, Valverius P (1985). 5Hydroxyindoleacetic acid in cerebrospinal fluid in alcoholic patients under different clinical conditions. Alcohol 2: 415-418.

Brown GL, Ebert MH, Goyer PF, Jimerson DC, Klein WJ, Bunney WEJ et al (1982). Aggression, suicide, and serotonin: relationships to CSF amine metabolites. Am J Psychiatry 139: 741-746.

Brown GL, Goodwin FK, Ballenger JC, Goyer PF, Major LF (1979). Aggression in humans correlates with cerebrospinal fluid amine metabolites. Psychiatry Res 1: 131-139.

de Waal F (1989). Peacemaking Among Primates, Vol. 5. Harvard University Press: Cambridge, MA.

Doudet D, Hommer D, Higley JD, Andreason PJ, Moneman R, Suomi SJ et al (1995). Cerebral glucose metabolism, CSF 5-HIAA levels, and aggressive behavior in rhesus monkeys. Am J Psychiatry 152: 1782-1787.

Fleming J, Mullen P, Sibthorpe B, Attelwell R, Bammer G (1998). The relationship between childhood sexual abuse and alcohol abuse in women - a case control study. Addiction 93: 1787-1798. 
Harlow H (1965). Sexual behavior in rhesus monkeys. In: Beach $\mathrm{F}$ (ed). Sex and Behavior, Vol. 1. Wiley \& Sons: New York. pp 234265.

Hasegawa T, Hiraiwa M (1980). Social interactions of orphans observed in free-ranging troop of Japanese monkeys. Folia Primatol 33: 129-158.

Hausfater G (1972). Intergroup behavior of free-ranging rhesus monkeys (Macaca mulatta). Folia Primatol 18: 78-107.

Higley JD (in press). Aggression in Old World primates. In: Maestripieri D (ed). Primate Psychology: The Mind and Behavior of Human and Nonhuman Primates.

Higley JD, Hasert MF, Dodson A, Linnoila M, Suomi SJ (1992a). Treatment of excessive alcohol consumption using the serotonin reuptake inhibitor Sertraline in a nonhuman primate model of alcohol abuse. Paper Presented at Research Society on Alcoholism, San Diego, CA, June 13-18.

Higley JD, King ST, Hasert MF, Champoux M, Suomi SJ, Linnoila M (1996a). Stability of interindividual differences in serotonin function and its relationship to aggressive wounding and competent social behavior in rhesus macaque females. Neuropsychopharmacology 14: 67-76.

Higley JD, Linnoila M, Suomi SJ (1994). Ethological contributions: experiential and genetic contributions to the expression and inhibition of aggression in primates. In: Hersen M, Ammerman RT, Sisson L (eds). Handbook of Aggressive and Destructive Behavior in Psychiatric Patients. Plenum Press: New York. pp 17-32.

Higley JD, Mehlman PT, Higley SB, Fernald B, Vickers J, Lindell SG et al (1996b). Excessive mortality in young free-ranging male nonhuman primates with low cerebrospinal fluid 5-hydroxyindoleacetic acid. Arch Gen Psychiatry 53: 537-543.

Higley JD, Mehlman PT, Poland RE, Taub DT, Suomi SJ, Linnoila M (1997). Aggression, social dominance, serotonin, and causal relationships. Biol Psychiatry 42: 306-307.

Higley JD, Mehlman PT, Poland RE, Taub DM, Vickers J, Suomi SJ et al (1996c). CSF testosterone and 5-HIAA correlate with different types of aggressive behaviors. Biol Psychiatry 40: 10671082.

Higley JD, Mehlman P, Taub D, Higley SB, Vickers JH, Suomi SJ et al (1992b). Cerebrospinal fluid monoamine and adrenal correlates of aggression in free-ranging rhesus monkeys. Arch Gen Psychiatry 49: 436-441.

Higley JD, Suomi SJ (1989). Temperamental reactivity in nonhuman primates. In: Kohnstamm GA, Bates JE, Rothbart MK (eds). Temperament in Childhood. John Wiley \& Sons: New York. pp 153-167.

Higley JD, Suomi SJ (1996). Effect of reactivity and social competence on individual responses to severe stress in children: investigations using nonhuman primates. In: Pfeffer CR (ed). Intense Stress and Mental Disturbance in Children. American Psychiatric Press, Inc.: Washington, DC. pp 1-69.

Higley JD, Suomi SJ, Linnoila M (1996d). A nonhuman primate model of type II alcoholism? Part 2. Diminished social competence and excessive aggression correlates with low cerebrospinal fluid 5-hydroxyindoleacetic acid concentrations. Alcoholism: Clin Exp Res 20: 643-650.

Hiroeh U, Appleby L, Mortensen PB, Dunn G (2001). Death by homicide, suicide, and other unnatural causes in people with mental illness: a population-based study. Lancet 358: 2110-2112.

Jonsson EG, Norton N, Gustavsson JP, Oreland L, Owen MJ, Sedvall GC (2000). A promoter polymorphism in the monoamine oxidase A gene and its relationships to monoamine metabolite concentrations in CSF of healthy volunteers. J Psychiatr Res 34: 239-244.

Kalin NH (1999). Primate models to understand human aggression. J Clin Psychiatry 60(Suppl 15): 29-32.

Kelley S (1998). Stress and coping behaviors of substance-abusing mothers. J Soc Pediatr Nurses 3: 103-112.
Koford CB (1965). Population dynamics of rhesus monkeys on Cayo Santiago. In: DeVore I (ed). Primate Behavior: Field Studies of Monkeys and Apes. Holt, Rinehart \& Winston: New York. pp 53-110.

Kruesi MJ, Swedo S, Leonard H, Rubinow DR, Rapoport JL (1990). CSF somatostatin in childhood psychiatric disorders: a preliminary investigation. Psychiatry Res 33: 277-284.

Kyes RC, Botchin MB, Kaplan JR, Manuck SB, Mann JJ (1995). Aggression and brain serotonergic responsivity: response to slides in male macaques. Physiol Behav 57: 205-208.

Lindburg DG (1971). The rhesus monkey in North India: an ecological and behavioral study. In: Rosenblum LA (ed). Primate Behavior: Developments in Field and Laboratory Research, Vol. 2. Academic Press: New York. pp 1-106.

Linnoila VM, Virkkunen M (1992). Aggression, suicidality, and serotonin. J Clin Psychiatry 53: 46-51.

Linnoila M, Virkkunen M, Scheinin M, Nuutila A, Rimon R, Goodwin FK (1983). Low cerebrospinal fluid 5-hydroxyindoleacetic acid concentration differentiates impulsive from nonimpulsive violent behavior. Life Sci 33: 2609-2614.

Manuck SB, Flory JD, McCaffery J, Matthews K, Mann J, Muldoon M (1998). Aggression, impulsivity, and central nervous system serotonergic responsivity in a nonpatient sample. Neuropsychopharmacology 19: 287-299.

Mason W (1960). The effects of social restriction on the behavior of rhesus monkeys: I. Free social behavior. J Comp Physiol Psychol 53: 582-589.

Mehlman PT, Higley JD, Faucher I, Lilly AA, Taub DM, Suomi S et al (1994). Low CSF 5-HIAA concentrations and severe aggression and impaired impulse control in nonhuman primates. $\mathrm{Am} \mathrm{J}$ Psychiatry 151: 1485-1491.

Mehlman P, Higley JD, Faucher I, Lilly AA, Taub DM, Vickers JH et al (1995). Correlation of CSF 5-HIAA concentration with sociality and the timing of emigration in free-ranging primates. Am J Psychiatry 152: 907-913.

Mehlman PT, Higley JD, Fernald BJ, Sallee FR, Suomi SJ, Linnoila M (1997). CSF 5-HIAA, testosterone, and sociosexual behaviors in free-ranging male rhesus macaques in the mating season. Psychiatry Res 72: 89-102.

Mehlman PT, Westergaard GC, Hoos BJ, Sallee FR, Marsh S, Suomi SJ et al (2000). CSF 5-HIAA and nighttime activity in freeranging primates. Neuropsychopharmacology 22: 210-218.

Mitchell G (1968). Attachment differences in male and female infant monkeys. Child Dev 39: 611-620.

Moss HB (1987). Serotonergic activity and disinhibitory psychopathy in alcoholism. Med Hypotheses 23: 353-361.

Preuss U, Schuckit M, Smith T, Danko G (2002). A comparison of alcohol-induced and independent depression in alcoholics with histories of suicide attempts. J Stud Alcohol 63: 498-502.

Redmond DEJ, Katz MM, Maas JW, Swann A, Casper R, Davis JM (1986). Cerebrospinal fluid amine metabolites. Relationships with behavioral measurements in depressed, manic, and healthy control subjects. Arch Gen Psychiatry 43: 938-947.

Roy A, De JJ, Linnoila M (1989). Cerebrospinal fluid monoamine metabolites and suicidal behavior in depressed patients. A 5-year follow-up study. Arch Gen Psychiatry 46: 609-612.

Scheinin M, Chang WH, Kirk KL, Linnoila M (1983). Simultaneous determination of 3-methoxy-4-hydroxyphenylglycol, 5-hydroxyindoleacetic acid, and homovanillic acid in cerebrospinal fluid with high-performance liquid chromatography using electrochemical detection. Anal Biochem 131: 246-253.

Shively CA, Fontenot MB, Kaplan JR (1995). Social status, behavior, and central serotonergic responsivity in female cynomolgus monkeys. Am J Primatol 37: 333-340.

Soubrié P (1986). Reconciling the role of central serotonin neurons in human and animal behavior. Behav Brain Sci 9: 319-364.

Spencer-Booth Y (1968). The behavior of group companions towards rhesus monkey infants. Anim Behav 16: 541-557. 
Taub DM, Mehlman PT (1989). Development of the Morgan Island Rhesus Monkey colony. Puerto Rico Health Sci J 8: 159-169.

Virkkunen M, De Jong J, Bartko J, Linnoila M (1989). Psychobiological concomitants of history of suicide attempts among violent offenders and impulsive fire setters. Arch Gen Psychiatry 46: 604-606.

Virkkunen M, Kallio E, Rawlings R, Tokola R, Poland RE, Guidotti A et al (1994a). Personality profiles and state aggressiveness in Finnish alcoholic, violent offenders, fire setters, and healthy volunteers. Arch Gen Psychiatry 51: 28-33.
Virkkunen M, Rawlings R, Tokola R, Poland RE, Guidotti A, Nemeroff C et al (1994b). CSF biochemistries, glucose metabolism, and diurnal activity rhythms in alcoholic, violent offenders, fire setters, and healthy volunteers. Arch Gen Psychiatry 51: 20-27.

Westergaard GC, Mehlman PT, Shoaf SE, Suomi SJ, Higley JD (1999). CSF 5-HIAA and aggression in female primates: species and interindividual differences. Psychopharmacology 146: 440446.

Zuckerman M (1985). Sensation seeking, mania, and monoamines. Neuropsychobiology 13: 121-128. 